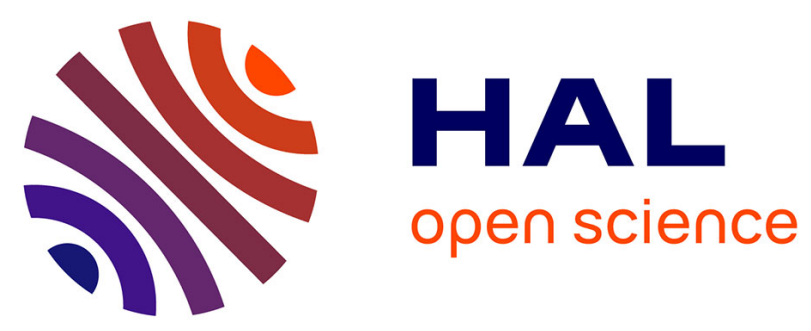

\title{
Contact laws between nanoparticles: the elasticity of a nanopowder
}

Adrien Girard, Julien Ramade, Jeremie Margueritat, Denis Machon, Lucien Saviot, Frédéric Demoisson, Alain Mermet

\section{To cite this version:}

Adrien Girard, Julien Ramade, Jeremie Margueritat, Denis Machon, Lucien Saviot, et al.. Contact laws between nanoparticles: the elasticity of a nanopowder. Nanoscale, 2018, 10 (4), pp.2154-2161. $10.1039 / \mathrm{c} 7 \mathrm{nr} 07540 \mathrm{e}$. hal-02515257

\section{HAL Id: hal-02515257 \\ https://hal.science/hal-02515257}

Submitted on 22 Oct 2020

HAL is a multi-disciplinary open access archive for the deposit and dissemination of scientific research documents, whether they are published or not. The documents may come from teaching and research institutions in France or abroad, or from public or private research centers.
L'archive ouverte pluridisciplinaire HAL, est destinée au dépôt et à la diffusion de documents scientifiques de niveau recherche, publiés ou non, émanant des établissements d'enseignement et de recherche français ou étrangers, des laboratoires publics ou privés. 

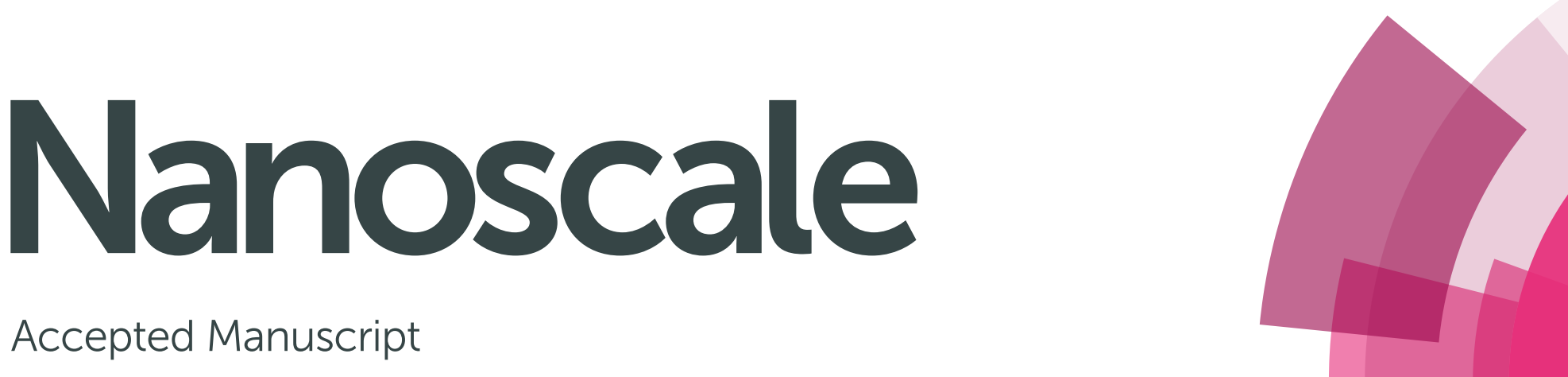

\section{Accepted Manuscript}

This article can be cited before page numbers have been issued, to do this please use: A. Girard, J. ramade, J. Margueritat, D. Machon, L. SAVIOT, F. DEMOISSON and A. Mermet, Nanoscale, 2018, DOI:
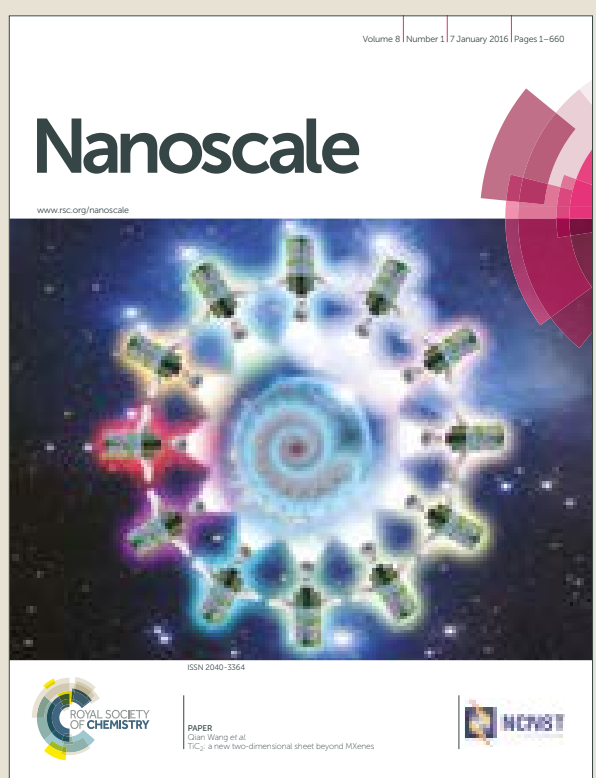

This is an Accepted Manuscript, which has been through the Royal Society of Chemistry peer review process and has been accepted for publication.

Accepted Manuscripts are published online shortly after acceptance, before technical editing, formatting and proof reading. Using this free service, authors can make their results available to the community, in citable form, before we publish the edited article. We will replace this Accepted Manuscript with the edited and formatted Advance Article as soon as it is available.

You can find more information about Accepted Manuscripts in the author guidelines.

Please note that technical editing may introduce minor changes to the text and/or graphics, which may alter content. The journal's standard Terms \& Conditions and the ethical guidelines, outlined in our author and reviewer resource centre, still apply. In no event shall the Royal Society of Chemistry be held responsible for any errors or omissions in this Accepted Manuscript or any consequences arising from the use of any information it contains. 


\section{Journal Name}

\section{ARTICLE TYPE}

Cite this: DOI: $10.1039 / \operatorname{xxxxxxxxxx}$

\section{Contact laws between nanoparticles: the elasticity of a nanopowder ${ }^{\dagger}$}

\author{
Adrien Girard, ${ }^{a}$, Julien Ramade, ${ }^{a}$, Jérémie Margueritat, ${ }^{a}$ Denis Machon, ${ }^{a}$ Lucien \\ Saviot, ${ }^{b}$ Frédéric Demoisson, ${ }^{b}$ and Alain Mermet ${ }^{a *}$
}

Received Date

Accepted Date

DOI: 10.1039/xxxxxxxxxx

www.rsc.org/journalname

\section{Introduction}

The mechanical properties of granular matter are of fundamental interest as they are widely spread and used both in industrial and geophysical contexts ${ }^{1,2}$. They are often addressed within effective medium approaches such as the Hertz-Mindlin theory ${ }^{3}$ whose validity has been thoroughly discussed in the case of micrometric beads packings ${ }^{4-9}$. The physics of inter-grain contact dynamics in such packings is usually addressed using acoustical methods such as ballistic propagation ${ }^{10}$ and multiple scattering of acoustic waves ${ }^{11,12}$. Lately, a new challenge has been to extend the validity of these contact laws down to the nanoscale ${ }^{13,14}$ where the mechanical properties stem from interactions between nano-surfaces ${ }^{15}$. In this case, spectroscopic techniques such as pump-probe experiments have been used to address the importance of the chemical state of the surfaces in the elasticity of assemblies of interacting nanoparticles (NPs) and the nature of the inter-NP bonding ${ }^{16,17}$.

The mechanical behavior of nanosurfaces relies on two main aspects: the nanocontact dynamics (i.e. how a nanomaterial acquires its mechanical properties from the bulk and grainboundary regions) and the friction or nanorheology (i.e. how energy is dissipated from the relative motion of nano-interfaces). Both computational ${ }^{18-20}$ and experimental ${ }^{21,22}$ efforts are be-

${ }^{a}$ Institut Lumière Matière, Université de Lyon, Université Claude Bernard Lyon 1, UMR CNRS 5306, 69622 Villeurbanne, France

${ }^{b}$ Laboratoire Interdisciplinaire Carnot de Bourgogne, UMR 6303 CNRS Université de Bourgogne Franche-Comté, 9 Av. A. Savary, BP 47870, F-21078 Dijon Cedex, France $\dagger$ Electronic Supplementary Information (ESI) available. ing continuously devoted to understanding these interface effects at the nanoscale, with a special emphasis on the role of chemical bonds ${ }^{23}$ and molecular adsorbates ${ }^{24}$ down to the single ion scale ${ }^{25}$. Nevertheless, the experimental verification of contacts laws at the nano and sub-nanoscales remains very difficult due to deviatoric effects inherent to the destructive nature of the methods used. For this reason, molecular dynamics (MD) simulations have been extensively used and have demonstrated a quantitative agreement between contact pressures and shear strengths ${ }^{19,26}$. These models capture the deformation at the nanoscale and show that roughness theories correctly describe the physics of deformation at the nanoscale. The question of heat transport across nano-interfaces through the propagation of heat-carrying phonons was also addressed theoretically ${ }^{27}$ and experimentally ${ }^{28}$ showing that thermal conductance is quantized across individual atomic-scale contacts within the contact zone. While MD-simulations are a powerful tool to theoretically investigate contact laws at the nanoscale, they deal with ideal systems with well-controlled surface states. Determining how these laws, i.e., contact elasticity and contact friction, are modified in real nanosystems with a high number of interacting objects and imperfect surface states remains a challenging issue.

In this paper, we show that in-situ Brillouin spectroscopy from thermal acoustic phonons in a nanopowder submitted to high hydrostatic pressures up to $16 \mathrm{GPa}$ can be used to characterize the contact dynamics and friction between the NPs. We show that the strongly non-linear behavior of the Brillouin frequency as a function of the applied pressure, long thought to arise from porosity and particle size variations, unambiguously arise from the NP 


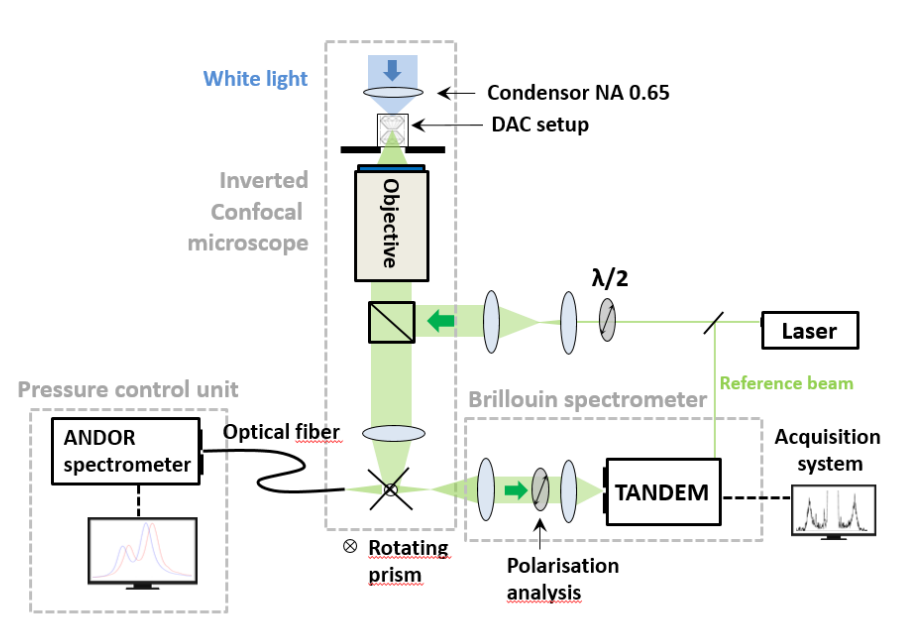

Fig. 1 Schematic view of the micro-Brillouin setup combined with the high pressure DAC setup, the pressure control unit equipped with an Andor spectrometer, the confocal microscope, and the Brillouin spectrometer.

(NP) contact dynamics as described by Hertz-Mindlin (HM) theory. The phonon damping due to inter-grain frictional dissipation can be also monitored from the Brillouin linewidth. In particular, we discuss the effects of the presence of organics at the NP surface, which strongly modifies both the powder elasticity and contact laws. These results are of fundamental interest as an experimental method of choice to investigate at the nanoscale in a non-destructive way both the nano-mechanical properties in the elastic approximation and nano-friction simultaneously.

\section{Experimental section}

$\mathrm{TiO}_{2}$ and $\mathrm{ZrO}_{2}$ nanopowders were prepared with a continuous hydrothermal production process under supercritical conditions previously described ${ }^{29,30}$. The diameter of the NPs is $3.6 \mathrm{~nm}$ for $\mathrm{TiO}_{2}$ and $5 \mathrm{~nm}$ for $\mathrm{ZrO}_{2}$ (standard deviation $1 \mathrm{~nm}$ ). The nanopowders were loaded in a diamond anvil cell (DAC) without transmitting medium together with a small ruby for pressure monitoring. The powders were placed in a $100 \mu \mathrm{m}$ hole drilled in a stainless gasket inside the diamond anvil cell to perform the Brillouin scattering measurements.

Micro-Brillouin experiments were carried out with a homemade setup presented in figure 1. It combines a tandem Fabry Perot interferometer equipped with an avalanche photodiode with an inverted confocal microscope and a pressure control unit. The Brillouin spectrometer is operated with the $532 \mathrm{~nm}$ wavelength from a solid state laser with power not greater than $10 \mathrm{~mW}$ to avoid damaging the sample. The measurements were done in the backscattering geometry with a long working distance $\times 20$ objective that was used for the in-situ DAC measurements. The light backscattered from the sample can be directed either towards the Brillouin spectrometer or the pressure control unit via a rotating prism. The pressure in the DAC cell is monitored using ruby fluorescence signal collected by the pressure control unit composed of a collecting optical fiber connected to an Andor spectrometer. The polarization can be controlled with a half-wave

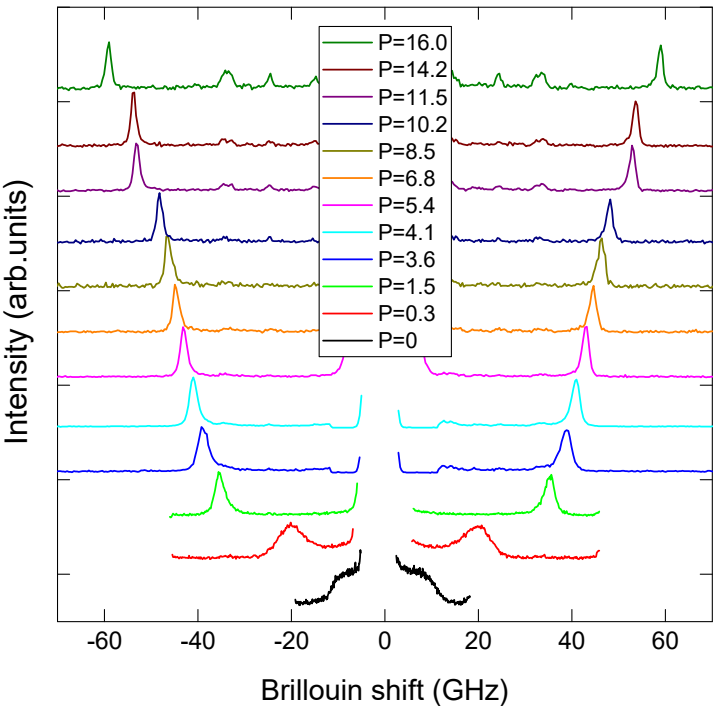

Fig. 2 Brillouin spectra of $\mathrm{ZrO}_{2}$ nanopowder as a function of applied pressure. The spectra are vertically shifted and the Rayleigh line has been omitted for clarity.

plate and is analyzed at the output of the confocal microscope with a grid polarizer.

\section{Results and discussion}

Brillouin spectroscopy of the nanopowders at high pressure. The Brillouin spectra of compressed $\mathrm{ZrO}_{2}$ powders at pressures up to $16 \mathrm{GPa}$ are shown in figure 2. The Brillouin spectra for the $\mathrm{TiO}_{2}$ nanopowders are provided as supplementary material. At ambient pressure a broad peak is observed in the foot of the Rayleigh line for both powders. It corresponds to the longitudinal Brillouin mode of the weakly compacted NPs. Upon pressure increase, the longitudinal Brillouin mode sharpens and shifts to higher frequencies. In the following we consider the results obtained with $\mathrm{ZrO}_{2} \mathrm{NPs}$, prior to discussing the results obtained with $\mathrm{TiO}_{2}$. The calculated sound velocity of the $\mathrm{ZrO}_{2}$ powder as a function of the pressure is shown in figure $3 \mathrm{~A}$ and $3 \mathrm{~B}$ with logarithmic scales. The observed behavior is highly non-linear, and the logarithmic representation shows that the sound velocity behaves as a power law of the applied pressure $P$. Such a nonlinear behavior of the sound velocity was previously reported in the case of compressed $\mathrm{MgO}$ nanopowders ${ }^{31}$ where the elastic stiffness was discussed in terms of grain size and porosity. Here we adopt a different approach to explain the powder elasticity in terms of the stiffness of inter-NP contacts based on HM theory, an effective medium theory that describes the mechanical deformation of compressed elastic spheres within the framework of continuum mechanics ${ }^{3}$. While this theory has been widely used to address the elasticity of compressed micrometric grains ${ }^{4-8}$, in particular for the description of the propagation of seismic waves in uncemented sediments (see ref 1 and references therein), it has not been tested to the best of our knowledge in the case of compressed NPs with sub-nanometric contact surfaces. 

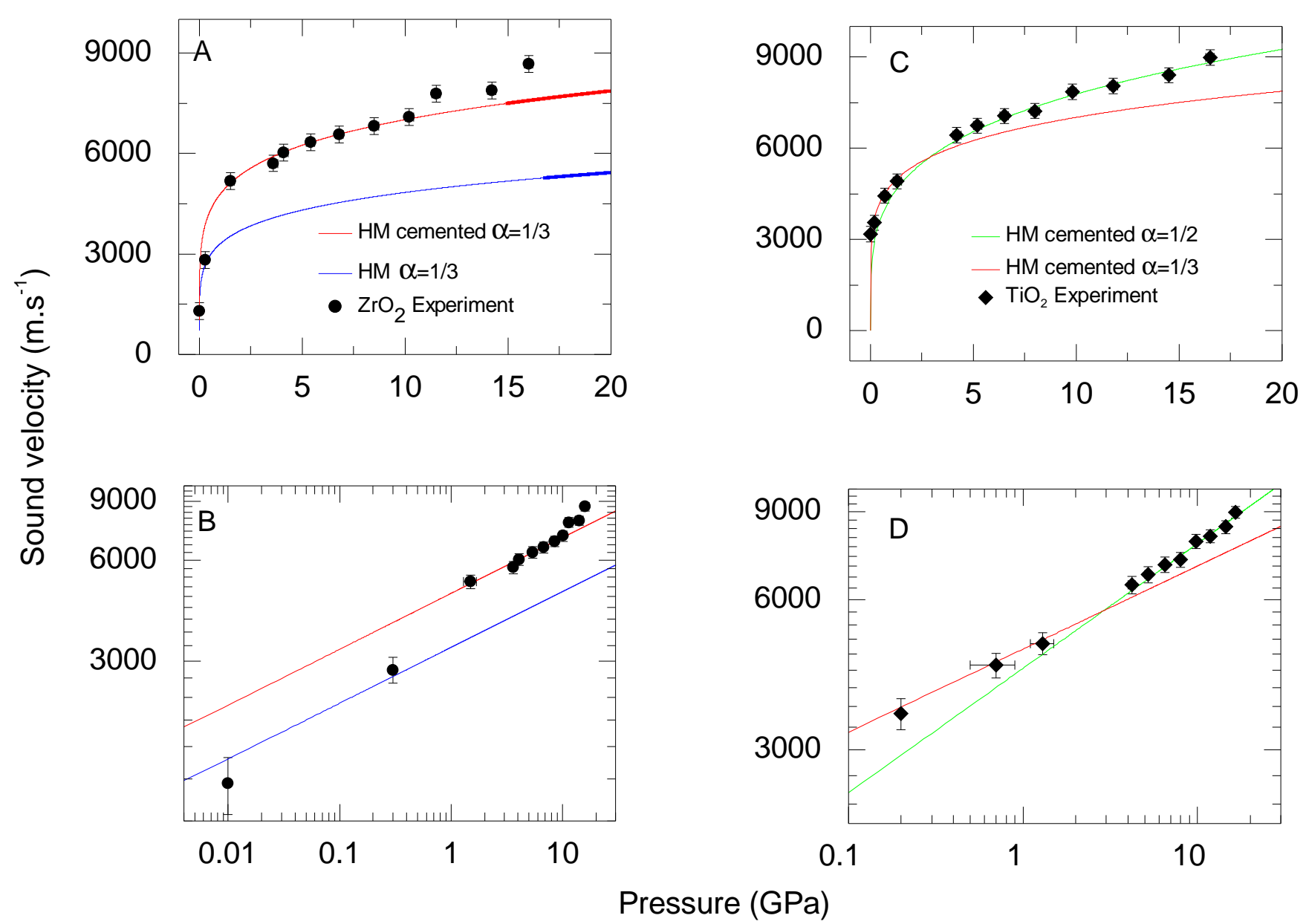

Fig. $3 \mathrm{~A}$ and $\mathrm{B}$ : Linear and logarithmic representation of the pressure dependence of the longitudinal acoustic velocity in $\mathrm{ZrO}_{2}$ nanopowder. Blue line is the result of HM theory when NPs have a bare surface and red lines correspond to the case where the NPs are cemented. C, D: Same for TiO ${ }_{2}$, with green line for cemented HM with $\alpha=1 / 2$ and red line for cemented HM with $\alpha=1 / 3$. 
Hertz Mindlin theory of the contact. Within the framework of HM theory, the longitudinal sound velocity is expressed as:

$$
V_{L}^{2}=\frac{2 C}{20 \pi R \rho}\left(S_{n}+\frac{2}{3} S_{\tau}\right)
$$

where $C$ is the coordinance, $R$ the NP radius, $\rho$ the mass density of the material and $S_{n}$ and $S_{\tau}$ the normal and tangential grain stiffnesses proportional to the inter-NPs contact radius $a_{\mathrm{HM}}$ (see supplementary info for details).

$$
\begin{aligned}
& S_{n}=\frac{4 \mu_{0} a_{\mathrm{HM}}}{1-v_{0}} \\
& S_{\tau}=\frac{8 \mu_{0} a_{\mathrm{HM}}\left(1-F_{t} / \eta F_{n}\right)^{1 / 3}}{2-v_{0}}
\end{aligned}
$$

where $v_{0}, \mu_{0}$ are the Poisson's ratio and the shear modulus of the NPs, $\eta$ is the coefficient of friction between the spheres and $F_{n}, F_{t}$ are the normal and tangential forces applied to the spheres respectively. This model considers that slip may occur at the edges of the contact, the extent of which depends on $\eta$. Here we are concerned with the propagation of longitudinal acoustic phonons. Therefore the normal force, due to the confining stress imposed on the NPs, is much larger than the tangential force, imposed by the oscillatory stress due to the acoustic wave propagation. Consequently, $F_{n} \gg F_{t}$, leading to a simplified expression of $\mathrm{S}_{\tau}$ :

$$
S_{\tau}=\frac{8 \mu_{0} a_{\mathrm{HM}}}{2-v_{0}}
$$

The dependence of the sound velocity on the applied pressure can be taken into account by considering small variations of contact area. Such small variations are estimated in the approximation of two interacting spheres by determining the pressure dependent radius of the contact area $a_{\mathrm{HM}}$ from HM theory ${ }^{3}$ :

$$
a_{\mathrm{HM}}(P)=R\left[\frac{3 \pi\left(1-v_{0}\right)}{2 C_{\max }\left(1-\phi_{\min }\right) \mu_{0}} P\right]^{\alpha}
$$

where $v_{0}$ and $\mu_{0}$ are the Poisson's ratio and the shear modulus of the material that constitutes the NPs, $C_{\max }=9$ is the coordinance number of a compacted disordered granular packing ${ }^{32}$, $\phi_{\min }$ the volume fraction of free space, and $\alpha=1 / 3$.

The calculated sound velocities are reported for $\mathrm{ZrO}_{2}$ in figure $3 \mathrm{~A}$ and $\mathrm{B}$, blue curves. Below $1 \mathrm{GPa}$ the dependence of the sound velocity as a function of the pressure is correctly reproduced using coordinance $\mathrm{C}=9$ and the minimum porosity for a disordered packing $\phi_{\min }=0.36$. However above $1 \mathrm{GPa}$, the absolute value of the sound velocity is underestimated by HM calculations. To explain this discrepancy, our first hypothesis was to consider the adhesive and frictional forces that arise at the nanoscale. These forces may be responsible for a stiffening of the inter-NPs contacts resulting in an increase of the sound speed $24,33,34$. Digby's approach allows to calculate these effects through a phenomenological model for two spheres initially bonded over a finite surface ${ }^{35}$. The results of the simulations applied to our oxyde nanopowders are provided as supplementary materials. Typically at very low applied pressures (in the MPa range, $\mathrm{P} \ll 1 \mathrm{GPa}$ ), the adhesion has a drastic effect on the sound speed due to the non-slipping

\begin{tabular}{ccc}
\hline & $\mathrm{TiO}_{2}$ & $\mathrm{ZrO}_{2}$ \\
\hline$\alpha=1 / 3$ & $\beta_{\text {cem }}=1.7$ & $\beta_{\text {cem }}=2$ \\
$\alpha=1 / 2$ & $\beta_{\text {cem }}=3.3$ & - \\
\hline
\end{tabular}

Table 1 Values of the fitting parameter $\beta_{\text {cem }}$ of the HM model for differents pressure exponents $\alpha=1 / 2$ and $\alpha=1 / 3$ corresponding to $\mathrm{HM}$ and soft shell model exponents respectively.

condition which increases drastically $V_{L}$. However at higher pressures, the effect is less pronounced and results essentially in a weaker additional offset to the sound speed compared to the nonbonded case. Overall this model does not reproduce our data indicating that the underestimation of the sound speed by HM theory does not arise from purely adhesive inter-NPs contacts.

Nanoparticle cementation and surface chemistry. Our oxyde NPs are produced in water and stored under ambient atmosphere. It has been evidenced that in such conditions their surface is covered by adsorbed $\mathrm{H}_{2} \mathrm{O}$ molecules. Water molecules are chemisorbed and additional molecules are physisorbed leading to a coverage of the nano-oxyde surface by about three $\mathrm{H}_{2} \mathrm{O}$ monolayers ${ }^{36}$. We have previously confirmed the presence of this water layer by evidencing its influence on the acoustic vibrations of $\mathrm{ZrO}_{2} \mathrm{NPs}^{37}$ from the same powder as the one presented here. In this latter case, the presence of a few water monolayers is at the origin of quasi-free acoustic vibrations due to the mechanical insulation even at high pressure. This makes it possible to consider an additional stiffening of the inter-grain elasticity, thereby affecting the nanopowder sound velocity. The effect of a small quantity of liquids on the elasticity of granular packs has been investigated previously ${ }^{38,39}$. The liquid in the intergrain region forms capillary bridges giving rise to additional adhesive forces, as well as lubrication of solid friction and viscous dissipation ${ }^{40}$, leading to an increase by a factor of 5 of the acoustic absorption ${ }^{41,42}$. Surface $\mathrm{H}_{2} \mathrm{O}$ molecules were recently shown to strongly influence the adhesion properties between oxyde $\mathrm{NPs}^{24}$. In particular, the molecular nature of adsorbed water molecules dictates the adhesion via solvation effects and influences the parameters of capillary models. However, at about $1 \mathrm{GPa}$ the liquid water-ice transition occurs and capillary forces and disjoining pressure effects are negligible. The solidification of the wetting liquid and compression of any extra molecules at the NPs contact may lead to an additional cementation effect that causes a large increase of the effective elastic stiffnesses of the material. In order to estimate how the inter-NPs cementation may influence our results, we used the cemented contact theory developed by Dvorkin et $\mathrm{al}^{43}$. This theory allows, in the case of small cement quantity, to estimate the effect of an additional elastic binder between the grains on the elasticity of an assembly of identical solid spheres ${ }^{8}$.

When the pressure is increased, the NPs are in contact through a finite surface area and the contact is no more punctual. The radius of the contact area between $\mathrm{ZrO}_{2}$ NPs can be calculated from HM theory (equation 5) and gives $a_{\mathrm{HM}} \sim 0.4 \mathrm{~nm}$ to $1 \mathrm{~nm}$ between 1 and $15 \mathrm{GPa}$. Following the formalism developed in ref 8 , the finite surface area of the contact was taken into account in the estimation of the cementation effect by introducing 


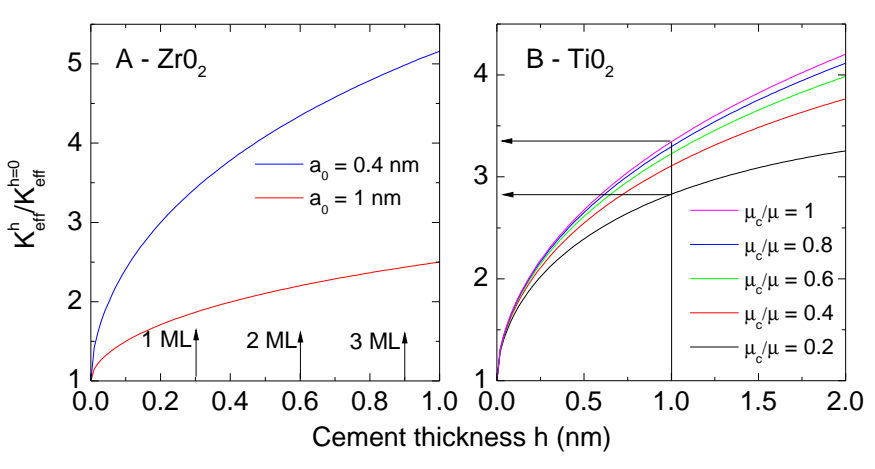

Fig. 4 A. Relative increase of the $\mathrm{ZrO}_{2}$ powder effective bulk modulus $\mathrm{K}_{\text {eff }}$ as a function of the thickness $h$ of a thin cementing layer possessing the elastic properties of bulk ice. Blue and red are for NP interpenetration radius of $0.4 \mathrm{~nm}$ and $1 \mathrm{~nm}$, corresponding to the $\mathrm{HM}$ radius values between 1 and $15 \mathrm{GPa}$ (probed pressure range). $\mathrm{B}$. Relative increase of the $\mathrm{TiO}_{2}$ powder $\mathrm{K}_{\text {eff }}$ versus cement thickness for various ratios of elastic modulii $\mu_{c} / \mu$ considering an initial NP interpenetration of $30 \%$, where $\mu_{c}$ is the elastic constant of the cement and $\mu$ is that of the NPs.

an initial contact radius $a_{0}$ in the cementation theory. Figure 4 A represents the relative increase of the effective bulk modulus of ice-cemented $\mathrm{ZrO}_{2}$ NPs as a function of the ice layer thickness $h$ for the two different initial bonding radii, corresponding to the $\mathrm{HM}$ radii of the NPs at 1 and $15 \mathrm{GPa}$, where capillary bridge solidification may exist. As shown in this figure one monolayer of ice $(0.3 \mathrm{~nm})$ is already sufficient to observe an increase of the elastic modulus by a factor $\sim 3$ at $1 \mathrm{GPa}$ and $\sim 2$ at $15 \mathrm{GPa}$. In order to take into account the cementation effect on the HM theory we have used $\left[S_{\mathrm{n}}, S_{\tau}\right]_{\text {cemented }}=\beta_{\text {cem }}\left[S_{\mathrm{n}}, S_{\tau}\right]_{\mathrm{HM}} \cdot \beta_{\text {cem }}$ is a scaling factor of the elastic modulus accounting for the cement. It is the only adjustable parameter of our model. The calculated sound velocities are reported for $\mathrm{ZrO}_{2}$ nanopowder in figure $3 \mathrm{~A}$ and $\mathrm{B}$ (red curves). The experimental sound velocities are well reproduced by HM-cemented model with $\beta_{\text {cem }}=2$ above $1 \mathrm{GPa}$, while the non-cemented model correctly reproduces the data at pressures lower than $1 \mathrm{GPa}$. This is in good agreement with the presence of liquid water - solid ice pressure transition occurring at about 1 GPa at ambient temperature and the relative increase of the stiffnesses predicted by the cementation. The adhesive effect of liquid water adsorbates is most likely too small to be observable in our experiments ${ }^{24}$.

Phase transitions. A significant deviation from our models is observed above $10 \mathrm{GPa}$. Bulk $\mathrm{ZrO}_{2}$ has three phase transitions upon pressure increase ${ }^{44}$ which are also observed in NPs powders ${ }^{37}$. It is thus suitable to determine how much the longitudinal sound velocity is sensitive to structural changes of the material the NPs are made of. The $\mathrm{ZrO}_{2}$ NPs have the monoclinic lattice structure at ambient pressure. They undergo two successive structural transitions towards an orthorhombic phase ortho I at about $6 \mathrm{GPa}$ and a second orthorhombic phase ortho II at about $11 \mathrm{GPa}$. No trace of the monoclinic-ortho I transition can be observed in the sound speed, presumably because of the weak elastic stiffness variation over this transition. However, a kink is detected above $11 \mathrm{GPa}$ after the NPs change to the ortho II lattice structure. The sound speed suddenly increases by $9.4 \%$, in good agreement with the relative stiffnesses of the two phases (monoclinic: $\mu_{0}=87 \mathrm{GPa}$, $v_{0}=0.29^{44}$ and ortho II $: \mu_{0}=115 \mathrm{GPa}, v_{0}=0.33^{44}$ ). This indicates that the Brillouin frequency of the nanopowder is sensitive to stiffness changes related to intra-NP phase transitions provided the variations are large enough compared to the contribution of the contact dynamics.

Surface chemistry: organic molecules. We have used the same formalism to evaluate the sound velocities in $\mathrm{TiO}_{2}$ powders and the results are also reported as red curves in figure $3 \mathrm{C}$ and $\mathrm{D}$. There is a good agreement with HM-cemented model for $P<2$ $\mathrm{GPa}$. However at higher pressure data diverges from the $\alpha=1 / 3$ power law. To explain the differences between $\mathrm{ZrO}_{2}$ and $\mathrm{TiO}_{2}$ we have to consider the production method of the nanopowders. In the case of $\mathrm{TiO}_{2}$ organic precursors are used for the synthesis, bis(ammonium lactato) titanium dihydroxide, resulting in lactate anions at the surface of the NPs. No organic precursors are used for $\mathrm{ZrO}_{2}$. Despite successive washing of the NPs, there are remaining organics at the surface of $\mathrm{TiO}_{2} \mathrm{NPs}$ as can be seen in the Raman spectra provided in supporting material. The strong $E_{g}$ mode at $144 \mathrm{~cm}^{-1}$ and three additional modes below $600 \mathrm{~cm}^{-1}$ are assigned to the anatase phase. The additional band at about $3000 \mathrm{~cm}^{-1}$ is not related to the anatase structure. It corresponds to the $\mathrm{C}-\mathrm{H}$ stretching frequency, ascertaining the coverage of the NPs by organic molecules. In other words there are not only water layers at the surface of the NPs but also some organics. If the amount of surface organics is sufficient to form a shell around the NPs, the powder elasticity may differ from the Hertz-like behavior. As predicted by De Gennes, a thin soft shell around solid beads induces an increase of the $\alpha$ parameter, i.e., the power law pressure dependence, from $1 / 3$ for free beads to $1 / 2$ for beads capped with an oxydation layer ${ }^{45}$. Using $\alpha=1 / 2$ and appropriate

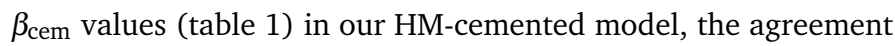
is satisfactory for pressure higher than $2 \mathrm{GPa}$, suggesting the importance of the surface chemistry of the NPs on the nanopowder elasticity. In this case there is no transition around $1 \mathrm{GPa}$ due to the water freezing since the organics presumably form a cement at ambient pressure, explaining why HM theory is not applicable even at low pressure. However the transition from $\mathrm{P}^{1 / 3}$ to $\mathrm{P}^{1 / 2}$ observed at relatively low pressures $(\mathrm{P} \sim 2 \mathrm{GPa})$ is probably due to a change in the elasticity of the organic layer. Indeed, for $\mathrm{ZrO}_{2}$, water molecules can be squeezed out of the contact area forming capillary bridges (no modification of Hertzian exponent). For $\mathrm{TiO}_{2}$ the organics presumably form a solid shell and remain within the contact area, leading to the soft shell effect $(\alpha=1 / 2)$.

Even though the elastic modulus of a surface organic layer is not known exactly, it is possible to estimate qualitatively the amplitude of the cementation effect when the organics are highly compacted. In figure $4 \mathrm{~B}$ we plot the relative increase of the $\mathrm{TiO}_{2}$ nanopowder bulk modulus $\mathrm{K}_{\text {eff }}$ versus the cement thickness for various ratios of the elastic modulii $\mu_{c} / \mu$ considering an initial NP interpenetration of $30 \%, \mu_{c}$ being the shear modulus of the 
cement and $\mu$ that of the NPs. Assuming for the sake of simplicity a $\sim 1 \mathrm{~nm}$ thick organic layer, the calculated scaling factor $2.8<\beta_{\text {calc }}<3.3$ for $0.2<\mu_{c} / \mu<1$ is in good qualitative agreement with the experimental one $\beta_{\mathrm{cem}}=3.3$. This is an important result of our study, where we show that the environment of the NPs and the nature of the adsorbates has a drastic influence on the powder mechanical properties. This interpretation is reminiscent of previous works that showed that the phase stability and the thermodynamics of the NPs can be tuned by controlling the NPs surface state ${ }^{46,47}$. Overall, they also provide additional insights into previous works on compressed nano-cristallites ${ }^{31,48}$, where the description of grain boundaries elasticity is rather discussed in terms of grain size and porosity evolution ${ }^{49-51}$. In our study, the independence of the NPs size with compression, as confirmed by previous low frequency Raman measurements 37,52 , ensures that the non-linearity of the sound velocity with the applied load stems from the contact dynamics.

The good agreement between experimental and calculated sound velocities suggests that we are probing the elastic regime even at very high pressures and that no plastic deformation occurs in the NPs. This assumption was experimentally verified through the monitoring of the NP Lamb mode frequencies, which are confined acoustic vibrations in the NPs, as a function of pressure. In a previous work, we have monitored the vibrations of individual NPs through the same sequence of pressure loading and unloading ${ }^{37}$. We have shown that pressure has no irreversible effect on the NPs Lamb frequencies because of the weak coupling between the NPs. A stronger coupling or plastic deformation would result in an hysteresis of Lamb frequencies or additional modes related to coupled NPs ${ }^{53}$. However the Brillouin frequency of the powder does not revert to the initial value after unloading both for $\mathrm{TiO}_{2}$ and $\mathrm{ZrO}_{2}$ (see supporting material). The hysteresis for the Brillouin frequency and the absence of hysteresis for the individual NPs acoustic vibrations is an indication that no plastic deformation occurs in the NPs in this pressure range. The Brillouin hysteresis is related to whether the adhesion between the NPs is very loose or very tight, which makes a big difference to the bulk sound velocity. Pressure application induces the NPs to be tightly forced together. After releasing the pressure, the NPs become highly glued together by either adsorbed water layers or organic compounds.

Frictional dissipation. In addition to the Brillouin frequency that gives access to the sound velocities, it is also possible to consider the Brillouin linewidth that is related to the mechanical damping of the vibrations, i.e., in our case to the attenuation of the elastic waves as a function of pressure. The damping is characterized by the attenuation factor $Q^{-1}=\delta v / v_{\text {peak }}$, where $v_{\text {peak }}$ is the frequency of the peak and $\delta v$ is its full width at half maximum. The values of $Q^{-1}$ for $\mathrm{ZrO}_{2}$ and $\mathrm{TiO}_{2}$ are reported in figure 5. We found that the acoustic attenuation decreases as a function of the applied pressure. To explain the origin of this mechanical damping we have used the Mindlin theory to determine numerically the attenuation factor $Q^{-1}$ due to the frictional and viscoelastic dissipation at the grain contacts :

$$
Q^{-1}=Q_{\text {vis }}^{-1}+Q_{\text {fric }}^{-1}
$$

where $Q_{\text {vis }}$ and $Q_{\text {fric }}$ are the quality factor due to the viscoelastic and frictional mechanisms respectively. The viscoelastic term $Q_{\mathrm{vis}}^{-1}$ contributes as a linear dissipation mechanism and is generally extracted from the measured data obtained in multiple sound scattering experiments ${ }^{42}$. In our case we consider that this term is small, and the attenuation factor can be thus expressed, in the approximation of smooth spheres, as a function of the shear displacement at the contact surface $U_{t}$, the pressure $P$ and the friction coefficient $\eta$, following the approach of ref 42 :

$$
Q^{-1} \approx Q_{\text {fric }}^{-1}=\left[\frac{4 \mu_{0}}{9 \pi^{2}\left(2-v_{0}\right) R}\left(\frac{3 \pi}{4 K_{0}}\right)^{1 / 3}\right] \eta^{-1} P^{-2 / 3} U_{t}
$$

where $K_{0}$ is the effective bulk modulus. For ultrasound experiments on micro-beads, the amplitude term $U_{t}$ is known. In our case, the value of $U_{t}$ for thermal phonons is not known precisely and the ratio $U_{t} / \eta$ is left as a fitting parameter. The results of the calculations are compared to the experimental $Q^{-1}$ value in figure 5 . The calculated values of $U_{t} / \eta$ are gathered in table 2 together with the corresponding values of $\eta$ assuming a displacement amplitude $U_{t} \sim 0.1 \AA$.

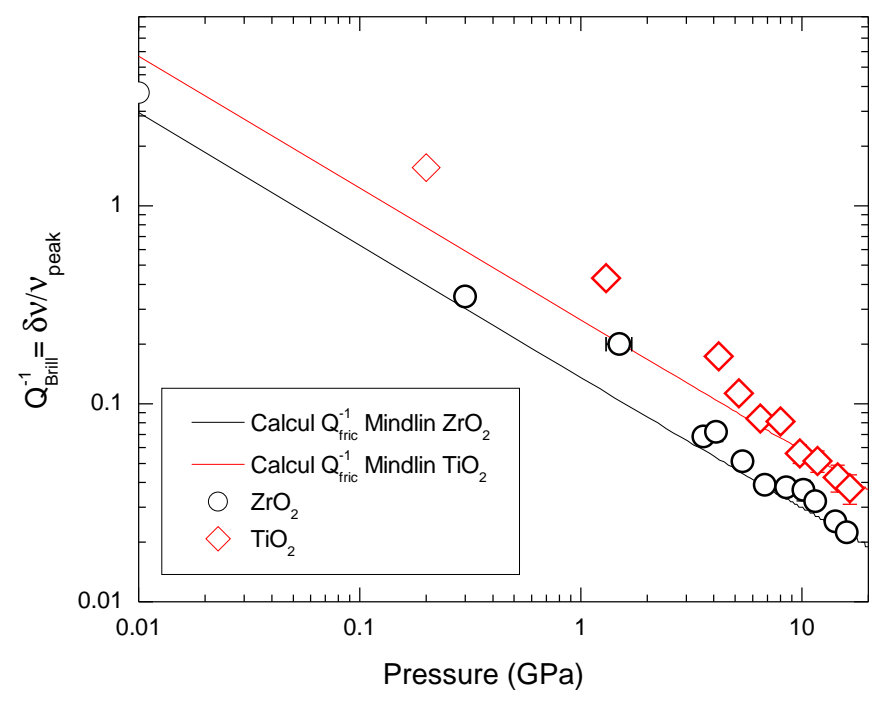

Fig. 5 Experimental values of $Q_{\mathrm{Brill}}^{-1}$ versus applied pressure $P$ for $\mathrm{ZrO}_{2}$ (black) and $\mathrm{TiO}_{2}$ (red) nanopowders deduced from Brillouin measurements compared to Mindlin calculations of the acoustic attenuation from the frictional mechanism $Q_{\text {fric }}^{-1}$ (full lines).

\begin{tabular}{ccc}
\hline & $\mathrm{TiO}_{2}$ & $\mathrm{ZrO}_{2}$ \\
\hline$U_{t} / \eta$ & 0.25 & 0.07 \\
$\eta$ & 0.4 & 1.5 \\
\hline
\end{tabular}

Table 2 Values of the calculated parameters $U_{t} / \eta$ of Mindlin model for the acoustic attenuation and corresponding values of $\eta$ considering a displacement amplitude $U_{t} \sim 0.1 \AA$. 
The good quantitative agreement suggests that the power law prediction for the acoustic energy dissipation is also valid for subnanometric contacts in the case of nanopowders. The smaller values of the friction coefficient for $\mathrm{TiO}_{2} \mathrm{NPs}$ are in line with the presence of residual organics as mentioned above, in agreement with previous reports in which the friction coefficient is reduced when measured on uncleaned bead surfaces ${ }^{42}$. The underestimation of $\mathrm{TiO}_{2}$ data by the theory below $5 \mathrm{GPa}$ can be explained by an additional source of viscous damping in the residual organics (not taken into account here) which disappears at highest pressures when organics are highly compacted.

\section{Conclusion}

We have shown that it is possible to quantitatively address the contact dynamics and the friction law in a nano-powder with high pressure Brillouin spectroscopy. The highly non-linear behavior of the compressed NPs is unambiguously attributed to the geometrical evolution of the contact area as described by the HM effective medium theory. Surface contamination by either water or organic molecules significantly alters the macroscopic elasticity. We assign this phenomenon to the modification of the contacts dynamics at the NP level. The Brillouin linewidth provides insights into the mechanisms of energy dissipation in the nanopowder, which stems primarily from nano-friction. These results suggest that macroscopic laws of elasticity and effective medium theories are relevant for the description of the mechanical properties of a nanopowder. This opens up perspectives in terms of characterization of nano-structured materials, such as the monitoring of the sintering processes in a nano-powder. These results can also benefit to geophysical sciences where high pressure Brillouin experiments are widely used to probe the elasticity of earth's mantle materials under extreme conditions of pressure and temperature ${ }^{54-57}$.

The authors thank gratefully Loic Vanel for fruitful discussions. This work was supported by the ANR NanoVip project, grant ANR.13.JSIO.0002 of the French Agence National de la Recherche and the Federation André Marie Ampère 2013 (FRAMA).

\section{References}

1 M. Saul, D. Lumley and J. Shragge, Geophysics, 2013, 78, D327-D338.

2 P. A. Johnson, H. Savage, M. Knuth, J. Gomberg and C. Marone, Nature, 2008, 451, 57-60.

3 J. D. G. Mavko, T. Mukerji, The Rock Physics Handbook, Second Edition, 2010.

4 C. Coste and B. Gilles, European Physical Journal B, 1999, 7, $155-168$.

5 B. Gilles and C. Coste, Physical Review Letters, 2003, 90, 174302.

6 J. D. Goddard, Proceedings of the Royal Society A: Mathematical, Physical and Engineering Sciences, 1990, 430, 105-131.

7 H. A. Makse, N. Gland, D. L. Johnson and L. M. Schwartz, Physical Review Letters, 1999, 83, 5070-5073.

8 V. Langlois and X. Jia, Physical Review E - Statistical, Nonlinear, and Soft Matter Physics, 2014, 89, 1-10.
9 X. Jia, C. Caroli and B. Velicky, Physical Review Letters, 1999, 82, 1863-1866.

10 S. Lherminier, R. Planet, G. Simon, L. Vanel and O. Ramos, Physical Review Letters, 2014, 113, 1-5.

11 R. L. Weaver and O. I. Lobkis, Physical Review Letters, 2001, 87, 134301.

12 M. L. Cowan, I. P. Jones, J. H. Page and D. A. Weitz, Physical Review E - Statistical, Nonlinear, and Soft Matter Physics, 2002, 65, 1-11.

13 B. Luan and M. O. Robbins, Nature, 2005, 435, 929-932.

14 S. Solhjoo and A. I. Vakis, Journal of Applied Physics, 2016, 120, 1-16.

15 X. Gao, F. Hao, Z. Huang and D. Fang, International Journal of Solids and Structures, 2014, 51, 566-574.

16 A. Ayouch, X. Dieudonné, G. Vaudel, H. Piombini, K. Vallé, V. Gusev, P. Belleville and P. Ruello, ACS Nano, 2012, 6, 10614-10621.

17 J. Avice, C. Boscher, G. Vaudel, G. Brotons, V. Juve, M. Edely, C. Méthivier, V. E. Gusev, P. Belleville, H. Piombini and P. Ruello, The Journal of Physical Chemistry C, 2017.

18 R. R. Santhapuram and A. K. Nair, Computational Materials Science, 2017, 136, 253-263.

19 Y. Mo, K. T. Turner and I. Szlufarska, Nature, 2009, 457, 1116-1119.

20 X. Liu, Z. Liu and Y. Wei, Computational Materials Science, 2015, 110, 54-61.

21 S. Tan, R. L. Sherman and W. T. Ford, Langmuir, 2004, 20, 7015-7020.

22 P. Paik, K. Kar, D. Deva and A. Sharma, Micro \& Nano Letters, 2007, 2, 90- 95.

23 K. Tian, N. N. Gosvami, D. L. Goldsby, Y. Liu, I. Szlufarska and R. W. Carpick, Physical Review Letters, 2017, 118, 1-6.

24 J. Laube, M. Dörmann, H.-J. Schmid, L. Mädler and L. Colombi Ciacchi, The Journal of Physical Chemistry C, 2017, 121, 15294-15303.

25 I. Counts, D. Gangloff, A. Bylinskii, J. Hur, R. Islam and V. Vladan, Phys. Rev. Lett., 2017, 119, 43601.

26 Y. Mo and I. Szlufarska, Physical Review B - Condensed Matter and Materials Physics, 2010, 81, 1-17.

27 B. Gotsmann and M. A. Lantz, Nature Materials, 2012, 12, 59-65.

28 S. de Mello, M. E. H. S. R. Maia da Costa, C. M. Menezes, C. D. Boeira, F. L. Freire Jr, F. Alvarez and C. A. Figueroa, Scientific Reports, 2017, 7, 3242.

29 C. Pighini, D. Aymes, N. Millot and L. Saviot, Journal of Nanoparticle Research, 2007, 9, 309-315.

30 F. Demoisson, M. Ariane and L. Saviot, Journal of Physical Chemistry C, 2011, 115, 14571-14575.

31 H. Marquardt, A. Gleason, K. Marquardt, S. Speziale, L. Miyagi, G. Neusser, Wenk and J. R, Mineralogical Magazine, 2011, 75, 1406.

32 W. O. Smith, P. D. Foote and P. F. Busang, Physical Review, 1929, 34, 1271-1274.

33 J. Laube, S. Salameh, M. Kappl, L. Mädler and L. Colombi 
Ciacchi, Langmuir, 2015, 31, 11288-11295.

34 S. Salameh, J. Schneider, J. Laube, A. Alessandrini, P. Facci, J. W. Seo, L. C. Ciacchi and L. Maedler, LANGMUIR, 2012, 28, 11457-11464.

35 P. J. Digby, Journal of Applied Mechanics, 1981, 48, 803.

36 A. V. Radha, O. Bomati-Miguel, S. V. Ushakov, A. Navrotsky and P. Tartaj, Journal of the American Ceramic Society, 2009, 92, 133-140.

37 L. Saviot, D. Machon, A. Mermet, D. B. Murray, S. Adichtchev, J. Margueritat, F. Demoisson, M. Ariane and M. D. C. Marco De Lucas, Journal of Physical Chemistry C, 2012, 116, 2204322050.

38 P. Tegzes, R. Albert, M. Paskvan, A. L. Barabasi, T. Vicsek and P. Schiffer, Physical Review E, 1999, 60, 5823-5826.

39 S. Nowak, A. Samadani and A. Kudrolli, Nat Phys, 2005, 1, 50-52.

40 J. Valenza, C. J. Hsu, R. Ingale, N. Gland, H. A. Makse and D. L. Johnson, Physical Review E - Statistical, Nonlinear, and Soft Matter Physics, 2009, 80, 51304.

41 X. Jia, Physical Review Letters, 2004, 93, 154303.

42 T. Brunet, X. Jia and P. Mills, Physical Review Letters, 2008, 101, 138001.

43 J. Dvorkin and A. Nur, Geophysics, 1996, 61, 890.

44 H. Ren, B. Zhu, J. Zhu, Y. Hao, B. Yu and Y. Li, Solid State Sciences, 2011, 13, 938-943.

45 P.-G. De Gennes, Europhysics Letters (EPL), 1996, 35, 145149.

46 D. Machon, M. Daniel, P. Bouvier, S. Daniele, S. Le Floch,
P. Melinon and V. Pischedda, The Journal of Physical Chemistry C, 2011, 115, 22286-22291.

47 L. Piot, S. L. Floch, T. Cornier, D. Machon, S. Le Floch, T. Cornier, S. Daniele and D. Machon, The Journal of Physical Chemistry C, 2013, 117, 11133-11140.

48 O. Yeheskel, R. Chaim, Z. Shen and M. Nygren, Journal of Materials Research, 2005, 20, 719-725.

49 H. S. Kim and M. B. Bush, Nanostructured Materials, 1999, 11, 361-367.

50 P. Sharma and S. Ganti, Journal of Materials Research, 2003, 18, 1823-1826.

51 J. Zhou, Y. Li, R. Zhu and Z. Zhang, Materials Science and Engineering A, 2007, 445-446, 717-724.

52 L. Saviot, D. Machon, L. Debbichi, A. Girard, J. Margueritat, P. Krager, M. C. Marco De Lucas and A. Mermet, Journal of Physical Chemistry C, 2014, 118, 10495-10501.

53 A. Girard, H. Gehan, A. Crut, A. Mermet, L. Saviot and J. Margueritat, Nano Letters, 2016, 16, 3843-3849.

54 M. Murakami, Y. Ohishi, N. Hirao and K. Hirose, Nature, 2012, 485, 90-94.

55 L. Dai, Y. Kudo, K. Hirose, M. Murakami, Y. Asahara, H. Ozawa, Y. Ohishi and N. Hirao, Physics and Chemistry of Minerals, 2013, 40, 195-201.

56 M. Murakami, S. V. Sinogeikin, H. Hellwig, J. D. Bass and J. Li, Earth and Planetary Science Letters, 2007, 256, 47-54.

57 M. Murakami, Y. Ohishi, N. Hirao and K. Hirose, Earth and Planetary Science Letters, 2009, 277, 123-129. 